\title{
Waste not, want not
}

\begin{abstract}
As of the writing of this Editorial, the current JCI Editorial Board has evaluated approximately 7,000 manuscripts over the past 22 months for their suitability for publication in our journal. While many of you have received a negative decision on your manuscript, I suspect few are aware of the changes we have made to our review process to limit reviewers' requests for what is in our view unnecessary and excessive experimentation.
\end{abstract}

Over the past few years, much has been written about the peer review process and reviewers' insistence on, along with journal editors' acquiescence to, numerous additional experiments as a condition of acceptance $(1,2)$. Whether this practice, which has evolved over time, has led to a better "product" is debatable. Indeed, if one uses reproducibility of published work as a metric of high-quality science, one can argue that we are failing miserably (3-5). So we must ask ourselves, what does it achieve to have a paper in the review process for 9-12 months or longer, undergoing multiple revisions and countless additional experiments that are then buried as on-line supplemental data? To me - not much!

I believe we have arrived at this point because of a convergence of a number of factors: First, there is a wealth of reagents and techniques available, essentially allowing a laboratory to perform almost any experiment imaginable, and, likewise, a reviewer who can request any imaginable experiment. Second, scientific malfeasance is all too common, and journal editors and the scientific community want and need the scientific record to be correct. Third, and I believe most important, many highprofile journals employ professional editors who are not actively engaged in scientific discovery to adjudicate decisions on manuscripts. Fortunately, the Editorial Board of the JCI functions differently. All the Associate Editors have active research programs, run their own research laboratories, submit their research discoveries to journals, and have experienced the despondency of a rejection letter and the excitement of a letter of acceptance. They have also been on the receiving end of an editor's unwillingness to overrule a reviewer's request for many additional and unnecessary experiments.

Because the JCI Editorial Board operates on a model in which scientists actively

Citation for this article: J Clin Invest. 2014; 124(2):463. doi:10.1172/JCI75011. engaged in scientific discovery are judging the science, we are in a position to reverse this damaging trend in science and the peer review process. Over the past few months, I have instructed the Board to intervene and not require authors to perform requested experiments if during the discussion of the manuscript the Associate Editor and Board believe the line of investigation is wasteful and not germane to the core message of the manuscript. But in order to accomplish a more streamlined review process, I will need your help.

To the authors: Your manuscript will be evaluated for what it is, not what we think it should become. If we do not feel your work provides a sufficient conceptual advance and/or lacks sufficient experimental rigor (6), we will tell you that it is best to submit your work elsewhere for publication. If, however, the Board determines that with the inclusion of some modest additional experimentation, the manuscript might reach our threshold for publication, we will follow the recommendations of the reviewers but also intervene if we determine that some of the experiments being requested are excessive.

To the reviewers: Limit your critique to the three most critical issues. There may be a few key experiments or additional controls that are needed to enhance the rigor and strength of the manuscript's conclusions. But evaluate the science that is in front of you. If, in your judgment, the submission lacks sufficient scientific rigor, or fails to sufficiently advance the field, tell the authors and us. However, we request that you not ask the authors to perform numerous experiments that move the study in new directions. Indeed, if after your thoughtful assessment of the science you are about to recommend the authors perform many additional experiments, then just reject the paper. Last, I believe timeliness of review is paramount for a successful review process. Therefore, we will be shortening the initial review period from 14 to 10 calendar days.
We at the JCI believe that we can make a difference and reverse this pernicious trend in science while still maintaining the highest standards of excellence at the journal and in the scientific literature. Having authors waste precious time and resources on experiments of limited value not only impedes the whole scientific endeavor, but also deflates the scientific spirit. We request that you work with us to provide timely critiques that move the review process in a new direction.

I, along with the rest of the Board, look forward to continuing to serve the JCI community and being on the leading edge of publishing new discoveries that advance our knowledge and change the practice of medicine. As always, I welcome your feedback (editors@the-jci.org).

\section{Howard A. Rockman, Editor in Chief}

1. Ploegh H. End the wasteful tyranny of reviewer experiments. Nature. 2011;472(7344):391.

2. Snyder SH. Science interminable: blame Ben? Proc Natl Acad Sci U S A. 2013;110(7):2428-2429.

3. Begley CG. Six red flags for suspect work. Nature. 2013;497(7450):433-434.

4. Begley CG, Ellis LM. Drug development: raise standards for preclinical cancer research. Nature. 2012;483(7391):531-533.

5. Prinz F, Schlange T, Asadullah K. Believe it or not: how much can we rely on published data on potential drug targets? Nat Rev Drug Discov. 2011; 10(9):712.

6. Henderson VC, Kimmelman J, Fergusson D, Grimshaw JM, Hackam DG. Threats to validity in the design and conduct of preclinical efficacy studies: a systematic review of guidelines for in vivo animal experiments. PLoS Med. 2013;10(7):e1001489. 